\title{
Tremor is a major feature of $9 p 13$ deletion syndrome
}

\author{
Susana Isabel Ferreira ${ }^{1}$ | Giacomo Cinnirella ${ }^{2}$ | Lina Ramos ${ }^{3}$ | Antonio Suppa ${ }^{4,5}$ | \\ Luís Miguel Pires $^{1}$ | Anna Maria Nardone ${ }^{6}$ | Letizia Camerota ${ }^{2}$ | Silvia Lanciotti ${ }^{2}$ | \\ Cinzia Galasso $^{7}$ | Fernando De Maio ${ }^{8}$ | Joana Barbosa de Melo ${ }^{1,9,10 ~ \mid ~}$ \\ Isabel Marques Carreira ${ }^{1,9,10}$ | Francesco Brancati ${ }^{11,12}$ \\ ${ }^{1}$ Laboratory of Cytogenetics and Genomics, Faculty of Medicine, University of Coimbra, Coimbra, Portugal \\ ${ }^{2}$ Medical Genetics Residency Program, Tor Vergata University of Rome and University of L'Aquila, L'Aquila, Italy \\ ${ }^{3}$ Medical Genetics Unit, Pediatric Hospital, Coimbra Hospital and University Centre, Coimbra, Portugal \\ ${ }^{4}$ Department of Human Neurosciences, Sapienza University, Rome, Italy \\ ${ }^{5}$ Neuromed IRCCS, Pozzilli, Isernia, Italy \\ ${ }^{6}$ Laboratory of Medical Genetics, Policlinico Tor Vergata, Rome, Italy \\ ${ }^{7}$ Department of Systems Medicine, Division of Child Neurology and Psychiatry, Tor Vergata University of Rome, Rome, Italy \\ ${ }^{8}$ Department of Orthopaedic Surgery, Tor Vergata University of Rome, Rome, Italy \\ ${ }^{9}$ Coimbra Institute for Clinical and Biomedical Research (iCBR), Area of Environment, Genetics and Oncobiology (CIMAGO), Faculty of Medicine, University of \\ Coimbra, Center for Innovative Biomedicine and Biotechnology (CIBB), Coimbra, Portugal \\ ${ }^{10}$ Clinical Academic Center of Coimbra (CACC), Coimbra, Portugal \\ ${ }^{11}$ Medical Genetics Laboratory, Department of Life, Health and Environmental Sciences, University of L'Aquila, L'Aquila, Italy \\ ${ }^{12}$ Istituto Dermopatico dell'Immacolata, IDI-IRCCS, Rome, Italy
}

\section{Correspondence}

Francesco Brancati, Medical Genetics, MeSVA Department, University of L'Aquila, Piazzale

Salvatore Tommasi, 1, 67100 Coppito,

L'Aquila, Italy.

Email: francesco.brancati@univaq.it

Funding information

Italian Ministry of Health, Grant/Award Numbers: Grant "Ricerca Finalizzata", Grant

"Ricerca Corrente"; University of L'Aquila, Grant/Award Number: Intramural funding "Bando FFO Ricerca 2020"

\begin{abstract}
Proximal interstitial deletions of chromosome 9p13 have been described only in a few patients with developmental delay, moderate intellectual disability, craniofacial dysmorphism, short stature, genital anomalies, and precocious puberty. To corroborate and expand these findings, we report on two novel syndromic male patients with 9 p13 deletions suffering from a similar form of tremor and compare them with literature data. Despite genomic variability in deletion sizes, all patients displayed homogeneous dysmorphism and clinical manifestations, including very invalidating tremor. Furthermore, we outlined a region of around $2 \mathrm{Mb}$ shared in common by all patients with nearly 70 genes, among which NPR2 might have a role in the phenotype. These data delineate interstitial 9p13 deletion syndrome with tremor as a major feature.

KEYWORDS

9p13 deletion syndrome, myoclonus, NPR2, tremor
\end{abstract}

\section{1 | INTRODUCTION}

Chromosomal deletion of distal 9p [OMIM \#158170] makes up a welldescribed clinical syndrome (Swinkels et al., 2008). Conversely, 9p proximal

Susana Isabel Ferreira and Giacomo Cinnirella contributed equally to this study. deletions are far less common with only a few cases published so far. In particular, two patients were diagnosed with 9p12p13 deletions at standard karyotyping (Eshel, Lahat, Reish, \& Barr, 2002; Giltay, Gerssen-Schoorl, \& van der Wagen, 1994), while in three distinct patients, variable deletions of 4.8-5.9 $\mathrm{Mb}$ were recently identified by genomic microarray analysis (Crone \& Thomas, 2016; Niemi, Kwan, Hudgins, Cherry, \& Manning, 2012). 
Despite the variability of deletion sizes, previous authors suggested the existence of a common phenotype mainly consisting of craniofacial dysmorphism, developmental and cognitive delay, precocious puberty, and abnormal genitalia (Crone \& Thomas, 2016). Of note, three of them had early-onset tremor (Crone \& Thomas, 2016; Niemi et al., 2012).

Here, we report on two novel patients with molecularly characterized 9p13 deletions narrowing down the smallest region of overlap (SRO) to $2.09 \mathrm{Mb}$. Their clinical features, together with those of previously reported cases, aid in the phenotypic delineation of this rare syndrome and outline tremor as a major clinical feature.

\section{2 | CLINICAL REPORT}

\subsection{Patient 1}

This 14-year-old boy was born at 35 weeks of gestation by cesarean delivery from a twin dizygotic pregnancy. Birth weight was $1,950 \mathrm{~g}$ (10th centile/small for gestational age), length $45 \mathrm{~cm}$ (25th centile), and head circumference $30.5 \mathrm{~cm}$ (10th centile). Mild hypotonia and difficulty in swallowing prompted feeding through parenteral nutrition. Developmental milestones were delayed: he stood with support at 24 months, walked at 30 , and said first words at 4 years. Bilateral cryptorchidism was surgically corrected. Occasionally, disruptive and oppositional behaviors, trichotillomania, and bruxism were noticed. In childhood, recurrent otitis media was registered but earing tested normal.

At age 14 years, he showed short stature $(153 \mathrm{~cm} ; 10$ th centile) with normal head circumference (56 cm; 70th centile) and craniofacial dysmorphism, including dolichocephaly, widow's peak, thick and laterally flared eyebrows, mild synophrys, prominent eyes with upslanted palpebral fissures, and long philtrum. Bilateral fifth fingers clinodactyly was noticed.

Neurological examination revealed abnormal speech and severe cognitive impairment, which precluded a detailed evaluation of the mental state. Cranial nerve function and eye movements were within normal ranges as well as motor and sensory systems. Deep tendon reflexes were present and symmetric in the upper as well as lower limbs. There was a flexor plantar reflex of the toe, bilaterally. Coordination of the limbs, gait, and balance appeared preserved. The main neurological symptoms pointed to a hyperkinetic disorder characterized by a mild resting and postural tremor prominently affecting the upper limbs and left side of the body. Resting and postural tremor were continuous/sub-continuous, rather irregular and variable but mostly characterized by high frequency and relatively small amplitude. Action tremor was also present in the upper limbs causing difficulties in skilled motor tasks such as drinking. Superimposed to tremor, he also manifested irregular jerky movements in the upper and, to a lesser extent, in the lower limbs, which are compatible with resting and action myoclonus. There were no clear features of reflex myoclonus. Bradykinesia/rigidity or ataxia was absent, and the rest of the neurologic examination was unremarkable.

\subsection{Patient 2}

This patient is the first child of healthy, unrelated parents, born at 38 weeks. Pregnancy was characterized by intrauterine growth restriction and hyperechogenic liver diagnosed at 29 weeks of gestation. His birth weight was $2,130 \mathrm{~g}$ (<third centile/small for gestational age), birth length $44.5 \mathrm{~cm}$ (<third centile), and head circumference $33 \mathrm{~cm}$ (10th centile). He was hypotonic and had intermittent hypoglycemic values in the first 2 days of life. Hepatic dysfunction was registered at birth with a raise in liver enzymes values and he underwent phototherapy. Liver ultrasound was normal, while hepatic biopsy showed moderate periportal hepatic steatosis and microvacuolar changes. At 6 months, he was hypotonic with growth difficulties. Severe difficulty in swallowing and chokes persisted until the end of the first year of life. The psychomotor delay was registered, and appropriate therapies started.

At the examination, aged 12 years, he presented dolichocephaly with prominent occiput, midface hypoplasia, and other dysmorphic features such as upslanted palpebral fissures, infraorbital creases, telecanthus and strabismus, asymmetric, and posteriorly rotated ears with prominent superior portion of the helix. Eyebrows were thick and wide with lateral flaring and mild synophrys. The nose was prominent with high nasal bridge and hypoplastic alae nasi; the philtrum was broad and tented (Figure 1a,b). Examination of the extremities demonstrated a single palmar crease in the right hand. He manifested short stature $(132 \mathrm{~cm}$, <third centile) with weight $31 \mathrm{~kg}$ (10th centile) and head circumference $52 \mathrm{~cm}$ (fifth centile). Hypoplastic genitalia were also noticed, without cryptorchidism. He suffered from atopic dermatitis and asthma. The language was delayed even having speech therapy since the age of 18 months. The parents referred that he used nonverbal communication several times (gestures and pointing) maintaining progress in this area. He had a global delayed psychomotor development with attention-deficit and hyperactivity disorder and Wechsler Intelligence Scale for Children-Third Edition score was 58. He underwent strabismus surgical correction. On neurological examination, he showed horizontal nystagmus that appeared in extreme eye movements. The coordination of limbs, gait, and balance was apparently preserved, except for dysmetria in the finger-nose test. It was worth mentioning the continuous presence of a slight tremor, affecting only the upper limbs and in a symmetrical way. The tremor was characterized mainly by high frequency and low amplitude and was exacerbated in situations of emotional tension or in the performance of tasks, many of which were impaired by it. Myoclonus was not present at rest or in action. The patient did not present bradykinesia or ataxia and the rest of the neurological examination was normal. Brain MRI showed mild hypoplasia of the corpus callosum. 


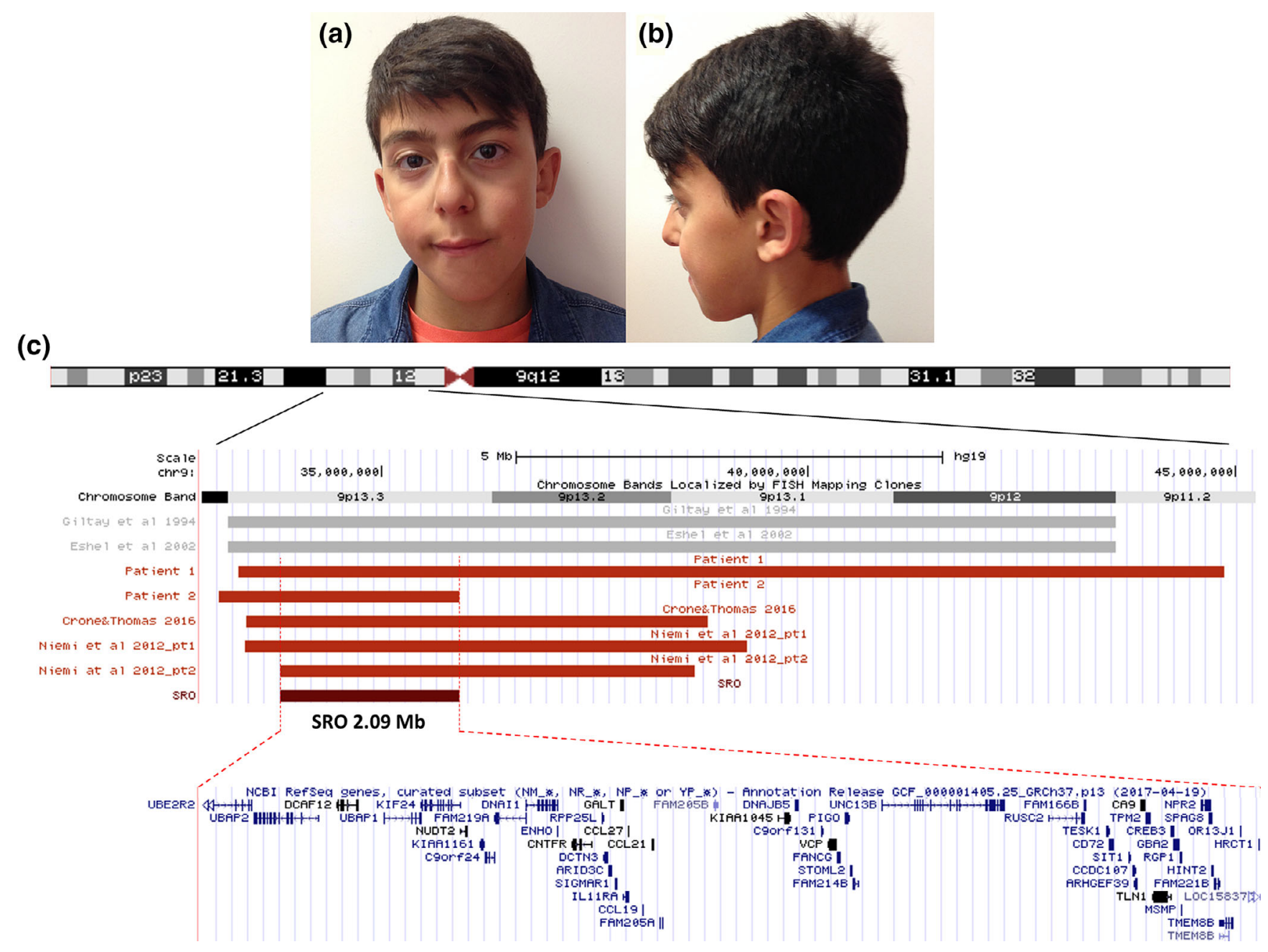

FIGURE 1 (a) Frontal and (b) lateral view of patient 2. Craniofacial dysmorphism include thick eyebrows, mild synophrys, prominent eyes and nose with high nasal bridge, asymmetric ears, midface hypoplasia, and dolichocephaly with prominent occiput. (c) Overview of the 9p13.3p11.2 chromosomal region: deletions identified at array-CGH in this and previous reports are in red, while patients diagnosed by cytogenetic analysis are shown in light gray. The smallest region of overlap (SRO) spanning about $2.09 \mathrm{Mb}$ at 9p13.3 is highlighted with its coordinates according to the UCSC Genome Browser (NCBI GRCh37/hg19) [Color figure can be viewed at wileyonlinelibrary.com]

\section{3 | METHODS AND RESULTS}

DNA from peripheral blood was extracted using standard methods and used to perform array comparative genomic hybridization (array-CGH) on a diagnostic basis using the Agilent SurePrint G3 Human Genome $180 \mathrm{~K}$ microarray (Agilent Technologies, Santa Clara, CA). Human genome build hg19/GRCh37 was used as a reference. Imbalances were interpreted with the use of the UCSC genome browser (http://genome. ucsc.edu), Decipher (http://decipher.sanger.ac.uk/), ClinGen (Clinical Genome Resource, https://www.clinicalgenome.org/), OMIM (Online Mendelian Inheritance in Man, http://www.ncbi.nlm.nih.gov), and DGV (http://projects.tcag.ca) databases. Fluorescence in situ hybridization analysis was performed with probe RP11-182N22 mapping to 9p13.3 labeled in red (BlueGnome, Illumina, San Diego, CA) to confirm the array-CGH result and verify the origin of the imbalance.

A $11.5 \mathrm{Mb}$ deletion spanning from $33,333,191$ to $44,870,148 \mathrm{bp}$ was identified in patient 1 , while patient 2 carried a deletion of about
$2.8 \mathrm{Mb}$ from $33,100,287$ to $35,911,318$ (Figure 1c). Both rearrangements arose de novo as postulated by segregation analysis in parental samples, which tested negative for the specific rearrangement. The identified deletions were compared to those described by Crone and Thomas (2016), Niemi et al. (2012), Giltay et al. (1994), and Eshel et al. (2002) thus defining the SRO shared by all patients extending $2.09 \mathrm{Mb}$ from $33,819,009$ to $35,911,318$ (Figure $1(C))$. This SRO included 76 genes, 14 of which are associated with at least one OMIM phenotype (Supplementary Table 1).

\section{4 | DISCUSSION}

The first report of a 9p12p13 deletion visible at conventional karyotyping is dated 1994 in a newborn female with developmental delay, dysmorphism, feeding difficulties, and external genitalia abnormalities, as seen at last examination aged 18 months (Giltay 
TABLE 1 Comparison of the clinical features observed in patients with 9p13 deletion syndrome

\begin{tabular}{|c|c|c|c|c|c|c|c|}
\hline & $\begin{array}{l}\text { Patient } 1 \\
\text { this report }\end{array}$ & $\begin{array}{l}\text { Patient } 2 \\
\text { this report }\end{array}$ & $\begin{array}{l}\text { Niemi et al. (2012) } \\
\text { Case } 1\end{array}$ & $\begin{array}{l}\text { Niemi } \\
\text { et al. (2012) } \\
\text { Case } 2\end{array}$ & $\begin{array}{l}\text { Crone and } \\
\text { Thomas (2016) }\end{array}$ & $\begin{array}{l}\text { Giltay } \\
\text { et al. (1994) }\end{array}$ & $\begin{array}{l}\text { Eshel } \\
\text { et al. }(2002\end{array}$ \\
\hline Gender & Male & Male & Female & Male & Female & Female & Female \\
\hline Cytogenetic band & 9p13.3p11.2 & $9 \mathrm{p} 21.1 \mathrm{p} 13.3$ & 9p13.3p13.1 & 9p13.3p13.1 & 9p13.3p13.1 & $9 p 12 p 13$ & $9 p 12 p 13$ \\
\hline Coordinates & $33.3-44.8$ & $33.1-35.9$ & $33.4-39.3$ & $33.8-38.6$ & $33.4-38.8$ & - & - \\
\hline Growth at birth & SGA & IUGR & IUGR & NR & NR & NR & SGA \\
\hline $\begin{array}{l}\text { Feeding } \\
\text { difficulties }\end{array}$ & + & + & - & - & + & + & - \\
\hline $\begin{array}{l}\text { Short stature } \\
\text { (centile) }\end{array}$ & $+(<10$ th $)$ & $+(<3 r d)$ & + & + & + & - & $+(5$ th $)$ \\
\hline Bruxism & + & + & + & - & + & NR & NR \\
\hline $\begin{array}{l}\text { Cognitive } \\
\text { impairment/ } \\
\text { developmental } \\
\text { delay }\end{array}$ & + & + & + & + & + & + & + \\
\hline $\begin{array}{l}\text { ADHD/behavioral } \\
\text { disorders }\end{array}$ & + & + & + & + & + & NA & + \\
\hline $\begin{array}{l}\text { Sleeping } \\
\text { difficulties }\end{array}$ & - & - & + & - & + & - & - \\
\hline Puberty & Normal & Normal & Precocious & Normal & Normal & NA & Precocious \\
\hline
\end{tabular}

Abbreviations: CCH: corpus callosum hypoplasia; DM: delayed myelination; IUGR: intrauterine growth retardation; MRI: magnetic resonance imaging; SGA: small for gestational age; NA: not applicable; NR: information not registered or unknown/not mentioned in the original report.

Note: When available, coordinates and sizes of deletions are given in megabases according to the Human Genome build hg19/GRCh37.

et al., 1994). In 2002, Eshel et al. described a second 9-year-old girl with 9p12p13.3 deletion associated with craniosynostosis, facial dysmorphism, ambiguous genitalia, developmental delay, attention-deficit hyperactivity disorder (ADHD), and precocious puberty.

Next, two distinct patients were reported by Niemi et al. (2012) with 9p13.3p13.1 deletions found at array-CGH and resulting in dysmorphic features, developmental delay, ADHD, and behavioral disorders. Interestingly, both also manifested an invalidating form of tremor. In the 16-year-old female, tremor of the hands started at 9 years of age and worsened with age, while myoclonic jerks were also present upon relaxation. In the other male patient, aged 7 and half years, tremor worsened under emotional stress. This association was confirmed by Crone and Thomas (2016) who described the fifth patient with 9p13.3p13.1 deletion manifesting tremor since the age of 3 years. It was postural and worsened during stressful activities or anxiety, again associated with early-onset myoclonic jerks, later resolved. Notably, the tremor was registered in all but two initially described patients, although we cannot exclude a later age of onset in them. Unfortunately, we could not further characterize this form of tremor by dedicated neurophysiological studies because the parents refused further instrumental investigations, but this would have been of importance to gain insights into the pathophysiology of this specific hyperkinetic disorder (Ure, Dhanju, Lang, \& Fasano, 2016).

In addition to tremor, we outlined several clinical manifestations easing the recognition of interstitial deletion 9p13 syndrome. All patients had developmental delays, and all but one showed behavioral disorders, mainly ADHD, but also aggressive behavior, hitting, vocalizations. Short stature, manifesting in $6 / 7$ patients, was a cardinal feature. Four patients had bruxism. Five showed genital anomalies, while precocious puberty was recorded only in two (Table 1). 
Recurrent otitis media was recorded in three patients. Notably, distinctive craniofacial dysmorphism emerged at comparative analysis (Supplementary Table 2). The cranial shape was often abnormal, mainly brachy but also dolichocephalic. One patient underwent early bifrontal craniotomy for craniosynostosis. Most had a highly arched palate. The ocular region was distinctive with prominent eyes, thick and laterally flared eyebrows, synophrys, upslanted palpebral fissures sometimes associated with epicanthal folds. The nose was prominent with a high nasal bridge, a long philtrum, and a thin upper lip. Ears were low-set and dysmorphic.

Our data also defined an interval of $2.09 \mathrm{Mb}$ as the SRO at 9p13.3 (Figure 1c) associated with a common syndromic phenotype containing nearly 70 genes (Supplementary Table 1). The large size of this SRO and the high number of genes make it difficult to conclude for distinct gene-to-phenotype correlations. Indeed, only the NPR2 gene seems to have an obvious role in the phenotype since its haploinsufficiency has been implicated in autosomal dominant idiopathic short stature (Wang et al., 2015). In fact, due to the very different sizes observed among reported deletions (Figure 1c) and the absence of obvious topologically associating domains (TADs) at the locus (data not shown), we reasoned haploinsufficiency could be considered as the most likely pathogenic mechanism. In addition, we cannot exclude that other noncoding elements at 9p13.3 might participate in the pathogenesis of the condition.

In conclusion, we outlined the clinical phenotype of chromosome 9p13 deletion syndrome with tremor as a cardinal manifestation. It is noteworthy that, in the absence of neurophysiological evidence of the cortical origin of tremor (e.g., back-averaging EEG), it is difficult to reach firm conclusions about the pathophysiology of this specific hyperkinetic disorder and further studies are encouraged to characterize this aspect. Additional investigations are also needed to identify the major genetic determinants of $9 \mathrm{p} 13$ deletion syndrome, paving the way to personalized treatment, especially for neurological manifestations.

\section{ACKNOWLEDGMENTS}

We thank Malte Spielmann for investigating possible TADs in the studied region. F.B. received funding from the Italian Ministry of Health, "Ricerca Finalizzata" and from the University of L'Aquila, "Bando FFO Ricerca 2020."

\section{CONFLICT OF INTEREST}

The authors declare no conflict of interest.

\section{AUTHOR CONTRIBUTION STATEMENT}

S.I.F. and G.C. wrote the first draft of the manuscript, tables, and figures. L.R., L.C., and S.L. performed the clinical evaluation and follow- up, reviewed the literature, and drafted the clinical description. A.S. performed neurological evaluation. C.G. and F.D.M. performed clinical, neuropsychiatric and orthopedic evaluations. A.M.N., L.M.P., and J.B.d.M. carried out, analyzed and interpreted cytogenetic data. I.M.C. and F.B. conceived and supervised the work, reviewed and verified all data, and finalized the manuscript. All authors discussed, read, and approved the final manuscript.

\section{DATA AVAILABILITY STATEMENT}

We confirm the absence of shared data

\section{ORCID}

Francesco Brancati (D) https://orcid.org/0000-0003-3624-2354

\section{REFERENCES}

Crone, M., \& Thomas, M. A. (2016). 9p13.1p13.3 interstitial deletion: A case report and further delineation of a rare condition. American Journal of Medical Genetics, Part A, 170A, 1095-1098.

Eshel, G., Lahat, E., Reish, O., \& Barr, J. (2002). Neurodevelopmental and behavioral abnormalities associated with deletion of chromosome $9 \mathrm{p}$. Journal of Child Neurology, 17, 50-51.

Giltay, J. C., Gerssen-Schoorl, K. B., \& van der Wagen, A. (1994). A case of de novo interstitial deletion of chromosome 9(p12p13). Clinical Genetics, 46, 271-272.

Niemi, A.-K., Kwan, A., Hudgins, L., Cherry, A. M., \& Manning, M. A. (2012). Report of two patients and further characterization of interstitial 9p13 deletion-a rare but recurrent microdeletion syndrome? American Journal of Medical Genetics, Part A, 158A, 2328-2335.

Swinkels, M. E. M., Simons, A., Smeets, D. F., Vissers, L. E., Veltman, J. A., Pfundt, R., ... van Ravenswaaij-Arts, C. M. A. (2008). Clinical and cytogenetic characterization of 13 Dutch patients with deletion $9 p$ syndrome: Delineation of the critical region for a consensus phenotype. American Journal of Medical Genetics, Part A, 146A, 1430-1438.

Ure, R. J., Dhanju, S., Lang, A. E., \& Fasano, A. (2016). Unusual tremor syndromes: Know in order to recognise. Journal of Neurology, Neurosurgery, and Psychiatry, 87, 1191-1203.

Wang, S. R., Jacobsen, C. M., Carmichael, H., Edmund, A. B., Robinson, J. W., Olney, R. C., ... Dauber, A. (2015). Heterozygous mutations in natriuretic peptide receptor-B (NPR2) gene as a cause of short stature. Human Mutation, 36, 474-481.

\section{SUPPORTING INFORMATION}

Additional supporting information may be found online in the Supporting Information section at the end of this article.

How to cite this article: Ferreira SI, Cinnirella G, Ramos L, et al. Tremor is a major feature of $9 \mathrm{p} 13$ deletion syndrome. Am J Med Genet Part A. 2020;182A:2694-2698. https://doi. org/10.1002/ajmg.a.61807 